\title{
Dynamic Analysis of Cardiac R-R Intervals in Normal Infants and in Infants Who Subsequently Succumbed to the Sudden Infant Death Syndrome
}

\author{
V. L. SCHECHTMAN, S. L. RAETZ, R. K. HARPER, A. GARFINKEL, A. J. WILSON, \\ D. P. SOUTHALL, AND R. M. HARPER
}

\begin{abstract}
Brain Research Institute and Department of Anatomy and Cell Biology, UCLA School of Medicine, Los Angeles, California 90024-1761 [V.L.S., S.L.R., R.K.H., A.G., R.M.H]; Department of Medical Physics and Clinical Engineering, Royal Hallamshire Hospital, Sheffield, United Kingdom [A.J.W.]; and National Heart and Lung Institute, London, United Kingdom [D.P.S.]
\end{abstract}

\begin{abstract}
Infants who subsequently succumb to the sudden infant death syndrome (SIDS) have higher heart rates and reduced heart rate variation compared with other infants. We examined dynamic changes in cardiac interbeat intervals to explore these differences in cardiac control. Recordings of electrocardiographic activity and respiratory movement were acquired from 13 SIDS victims before their deaths. Moment-to-moment changes in R-R intervals during quiet sleep, rapid eye movement sleep, and waking were compared with values of 13 matched control infants. For each sleep-waking state, every R-R interval was plotted against the previous interval (Poincaré plots), and each change in interbeat interval was plotted against the previous change. Dispersion of interbeat intervals at different heart rates was reduced in SIDS victims, resulting in Poincaré plots markedly different from those of controls. The dispersion, sampled at the 10th and 90th percentiles of heart rates, was reduced across all sleep-waking states in SIDS victims. At high heart rates, the difference between groups disappeared after correcting for basal rate; however, the reduced range at low heart rates was independent of basal rate. SIDS victims also showed smaller beat-to-beat changes in heart rate and fewer sustained runs of consistent heart rate changes during waking relative to controls. The differences in cardiac rate dynamics suggest altered autonomic control in infants who succumb to SIDS. We speculate that the autonomic disturbance may lead to cardiac instability or may indicate CNS alterations with the potential to affect other vital functions. (Pediatr Res 31: 606612, 1992)
\end{abstract}

Abbreviations

AW, waking

QS, quiet sleep

REM, rapid eye movement

SIDS, sudden infant death syndrome

ANOVA, analysis of variance

Infants who subsequently succumb to SIDS demonstrate higher heart rates, diminished heart rate variation associated with respiration, and disturbed long-term coordination of cardiac and

Received October 25, 1991; accepted February 6, 1992

Correspondence and reprint requests: Ronald M. Harper, Ph.D., Brain Research Institute, UCLA School of Medicine, Los Angeles, CA 90024-1761.

Supported by HD22695 from the National Institute of Child Health and Human Development, U.S. Public Health Service. respiratory measures relative to age-matched controls (1-5). Some of these differences are apparent only in particular sleepwaking states $(2,4,6)$. We examined respiratory patterning to determine the factors underlying the differences in heart rate variation and found fewer respiratory pauses in the SIDS victims (7); however, respiratory rate and its overall variability did not differ significantly from those of control infants (2). Despite the similarities on summary respiratory measures (respiratory rate and overall variability) between a group of infants who succumbed to SIDS and a group of infants who survived, some modulator of cardiac interbeat intervals must be responsible for the diminished cardiac variation observed in infants who later succumb to SIDS. Although summary measures of cardiac activity failed to distinguish those aspects, examination of momentto-moment dynamics of cardiac patterning may reveal the mechanisms influencing cardiac interval variation. Examination of dynamic aspects of cardiac interval changes reveal unique patterning in patients with congestive heart failure (8) and congenital central hypoventilation syndrome ["Ondine's Curse" (9)]; dynamic patterning of cardiac activity is also altered in animals with myocardial ischemia who succumb to sudden death (10) and after cocaine intoxication (11). We hypothesized that assessment of beat-to-beat aspects of cardiac interval changes would differentiate infants who later succumb to SIDS from matched control infants.

The procedures that detected the higher heart rates and diminished heart rate variation in infants at risk for SIDS yielded overall, or summary, characteristics of rate and changes in interbeat intervals over relatively long periods of time (measures of their central tendency). These procedures included spectral techniques, means, medians, SD, and interquartile ranges. To examine moment-to-moment, or dynamic, changes in cardiac intervals, we recruited techniques appropriate for assessment of beat-to-beat interval changes

A simple procedure, which provides information about both beat-to-beat and long-term changes in cardiac rate, involves plotting each $R-R$ interval (the interval between successive $R$ waves of the ECG) against the previous interval [Poincare plot (12)]. This procedure provides an indication of $l$ ) the average rate, 2) the dispersion in next-interval values for any observed heart rate, 3 ) the overall heart rate variation, which is reflected in the spread of values on both the $x$ and $y$ axes, and 4) the trend of dispersion in interbeat interval as rate changes. We adopted this procedure to examine patterns of cardiac variation in infants who later succumbed to SIDS and control infants.

To assess patterns of moment-to-moment change in heart rate, each $\Delta \mathrm{RR}$ (the time difference, measured in $\mathrm{ms}$, between two successive $\mathrm{R}-\mathrm{R}$ intervals, i.e. $\left.\mathrm{RR}_{n+2}-\mathrm{RR}_{n+1}\right)$ may be plotted against the previous difference $\left(R R_{n+1}-R R_{n}\right)$. Such a plot 
demonstrates the change in interbeat interval as a function of the previous interval change.

Patterns of heart rate variation are particularly sensitive to state influences; thus, sleep states must be controlled when using comparisons between SIDS victims and other infants. During QS, for example, short-term heart rate variation is enhanced and overall variance reduced relative to those values found in REM sleep (13). Furthermore, certain differences previously identified between SIDS victims and controls are apparent only during particular sleep-waking states $(2,4)$. We examined instantaneous and long-term changes in cardiac beat-to-beat intervals during sleep and AW states in control infants and infants who later succumbed to SIDS

\section{MATERIALS AND METHODS}

Subjects. Twenty-four-hour recordings of ECG and respiratory movements were obtained on one or two occasions from 6914 normal, full-term infants during the first few months of life (14). Sixteen of these infants later died with a postmortem determination of SIDS. Of these 16 infants, three subjects were excluded from analyses because excessive artifacts in their recordings precluded accurate sleep-state classification. For each of the 13 recordings of SIDS victims, one recording of a surviving infant of similar age, gender, and birth weight was selected as a matched control. $t$ tests showed no significant difference in the birth weights of the SIDS victims and the controls $(p>0.05)$, and Kruskal-Wallis nonparametric ANOVA indicated no significant difference in ages $(p>0.05) . \chi^{2}$ analysis demonstrated no significant difference in the proportion of males to females between the group of SIDS victims and the group of controls ( $p$ $>0.05$ ).

Recording Procedures. All recordings were acquired using a Medilog (Oxford Medical Systems, Oxford, UK) four-channel recorder. Recordings of the 13 SIDS victims and the 13 matched controls were later digitized on a PDP-11/73 computer and stored on digital tape. The ECG was fed to a Schmitt trigger, which produced a pulse for each $\mathrm{R}$ wave, and the resulting trigger event times were recorded with the other digitized signals. Pulses from a crystal-controlled clock were placed on one channel of the recorder; these pulses were later used to control the analogto-digital converter on the computer, and thus ensured against errors from tape speed variation.

State Classification. The marked effects that sleep states exert on respiratory rate and variability were used to develop a discriminant function to classify sleep-waking states in infants (15). This system was developed on 12-h nighttime (1900-0700 h) recordings of infants; thus, only the nighttime portions of these data were state classified and used in analyses.

Breath-to-breath intervals (the time in ms from one respiratory peak to the next) were determined from the respiratory signals with a resolution of $63 \mathrm{~ms}$, and median breath-to-breath intervals (a measure of respiratory rate) and interquartile range of breathto-breath intervals (a measure of respiratory variability) were determined for each 1 -min epoch. Median breath-to-breath intervals corresponding to respiratory rates $>110$ breaths/min or $<15$ breaths/min are usually the result of artifacts; therefore, epochs with these aberrant median breath-to-breath intervals were labeled "artifact-contaminated." The median breath-tobreath interval for an epoch is only minimally affected by apneic episodes, which are represented by single long intervals; thus, epochs were not identified as artifactual on the basis of apneic pauses.

Cardiac $\mathrm{R}-\mathrm{R}$ intervals were computed from $\mathrm{R}$ wave trigger event times with a resolution of $2 \mathrm{~ms}$. An automated routine was used to identify artifacts in the cardiac R-R intervals, and, where possible, to correct artifacts by recombination of adjacent R-R intervals (16). Those 1-min epochs with many artifacts $(>30$ artifacts or several artifacts that could not be corrected by recombination of intervals) were also labeled "artifact contaminated."
Epochs identified as artifact contaminated based on aberrant values for respiratory rate or variability or on artifacts in the ECG triggers were eliminated from the study before the sleep state classification, thus minimizing the effect of artifacts on sleep state classification.

Each 1-min epoch between 1900 and $0700 \mathrm{~h}$ was classified as QS, REM sleep, AW, or indeterminate state based on aspects of cardiac and respiratory rate and variability. The AW state was identified principally by rapid heart rate and substantial variability in respiratory rate, QS by regular respiration with slow heart rate and enhanced respiratory-related heart rate variation, and REM sleep by slow, irregular respiratory rate and relatively slow heart rate with large low-frequency oscillations. To allow for accurate identification of sleep-waking states despite considerable intersubject variation (e.g. respiratory rate is higher in some infants than in others), values for each measure were normalized over each recording without regard to other recordings. $\mathrm{Z}$ scores from one individual were used to classify each epoch in the recording of that individual. This automated infant sleep state classification system is capable of identifying sleepwake states with a level of accuracy similar to that of a trained observer using all physiologic signals (15).

The output of the state classification routine was a list of 720 state codes for each recording. Each code represented the sleep state of the infant during a 1-min epoch beginning at $1900 \mathrm{~h}$ and ending at $0700 \mathrm{~h}$. Five codes were used to indicate QS, REM sleep, AW, indeterminate state, and artifact contamination.

Analyses. Cardiac R-R intervals were plotted as a function of time over the entire recording period. For each recording, all R$\mathrm{R}$ intervals in all 1-min epochs identified as QS were stored in one computer file. Similar files were constructed for REM sleep and AW. All subsequent analyses were performed on these 78 files (26 subjects $\times$ three sleep-waking states). All analyses used repeated measures ANOVA, in which each SIDS victim was compared with a matched control; thus, the effects of postnatal age were controlled. Tests of sphericity (17) were applied to all interactions to assess the degree of dependence of the factors. When these tests revealed significant dependence, the HuynhFeldt adjustment (18) was used, and the adjusted probability values are reported.

Summary Measures. The means and SD of the R-R intervals were calculated for the three data files from each subject, and ANOVA was used to compare these measures of heart rate and variability in the pairs of SIDS victims and matched controls.

$R R_{\mathrm{n}+1}$ Versus $R R_{\mathrm{n}}$ (Poincaré) Plots. Each cardiac interval length was plotted on the $y$ axis against the previous value on the $x$ axis, resulting in an $R R_{n+1}$ verstus $R_{n}$ (Poincaré) plot, where the R-R interval is the time in ms from one $R$ wave of the ECG to the next, and $n$ is the interval number. Each plot illustrates $I$ ) the beat-to-beat dispersion (changes in $R_{n+1}$ at a given value of $R R_{n}$ ), which is reflected in the scatter of values on the $y$ axis for any value of $x ; 2$ ) the change in dispersion as values on the $x$ axis change; and 3) the overall range of $\mathrm{R}-\mathrm{R}$ intervals, which is reflected in the absolute extent of dispersion of points on both the $x$ and $y$ axes.

Pearson's $r$ coefficient was used to assess the correlation of R$\mathrm{R}$ intervals with the subsequent intervals. The resulting $r$ values for each infant in each state were subjected to a square root transform to normalize their distribution, and the resulting values were subjected to a two-way ANOVA to assess differences across sleep states and risk groups.

To assess the extent of dispersion of points in the Poincare plots and the shape of the plots, the range of $y$ values at the 10th and 90th percentile values for $x$ were calculated. Because the range can be profoundly affected by outliers, the points in the highest and lowest $10 \%$ were excluded from calculations of the range; thus, the range was defined as the time difference, in $\mathrm{ms}$, from the 10th to the 90 th percentile of $y$ values for a given $x$. A three-way ANOVA [risk group $\times$ sleep state $\times$ heart rate $(10$ or $90 \%)$ ] was performed on these values. 
$\Delta R R_{\mathrm{n}+1}$ Versus $\Delta R R_{\mathrm{n}}$ Plots. To assess changes in R-R intervals, we constructed $\Delta R R_{n+1}$ versus $\Delta R R_{n}$ plots. Each interval was subtracted from the next to obtain a difference value: $\Delta R R_{n+1}=$ $R R_{n+1}-R R_{n}$. Each difference value was then plotted against the previous difference, resulting in a $\Delta R_{n+1}$ versus $\Delta R R_{n}$ plot. The purpose for calculating these values was to remove the dominant characteristic apparent in the Poincare plots, i.e. the strong positive correlation between one interval and the next. Thus, the $\Delta R_{n+1}$ versus $\Delta R R_{n}$ plots reveal serial dependencies of cardiac accelerations and decelerations.

The plots of differences between R-R interval changes can be envisioned as values distributed on an area defined by four quadrants. Each quadrant indicates the direction of two consecutive changes in interval length (Fig. 1). The $\Delta R R_{n+1}$ versus $\Delta R R_{n}$ plots therefore demonstrate 1 ) the ratio of short- to longterm changes in heart rate and 2) the trends of variation in heart rate.

Ratio of high-frequency to low-frequency heart rate variation. High-frequency heart rate variation is characterized by many changes in the slope of the R-R interval time series (an increase in $\mathrm{R}-\mathrm{R}$ interval length followed by a decrease and vice versa). High-frequency variation is therefore reflected by many points in quadrants $a$ and $d$ of Figure 1 . Low-frequency heart rate variation, on the other hand, is characterized by many sequential increases in interval length and many sequential decreases, indicated by points in quadrants $b$ and $c$ of Figure 1 .

Trends of variation in heart rate. If changes from one interval to the next occurred randomly and independently, all quadrants would have an approximately equal number of values. If, on the other hand, heart rate tended to increase quickly (decreasing R$\mathrm{R}$ intervals) and decrease more slowly (increasing R-R intervals), there would be a greater number of intervals representing decreasing heart rates (increasing R-R intervals); thus, more points would fall in quadrants $a$ and $b$ than in $c$ and $d$ of Figure 1 . The opposite would be the case if heart rate decreased more quickly than it increased.

Each $\Delta R R_{n+1}$ versus $\Delta R R_{n}$ plot was quantified by the total number of points in each quadrant. Because imprecision in measurement introduces "noise" in the form of very small apparent changes in heart rate, only those changes in R-R duration greater than $4 \mathrm{~ms}$ were included for analysis. A common log transform was used to normalize the distribution of numbers of points in each quadrant across infants and sleep-waking states. A three-way ANOVA (SIDS risk $\times$ state $\times$ quadrant) was performed on these $\log _{10}$ values. To account for differences in the

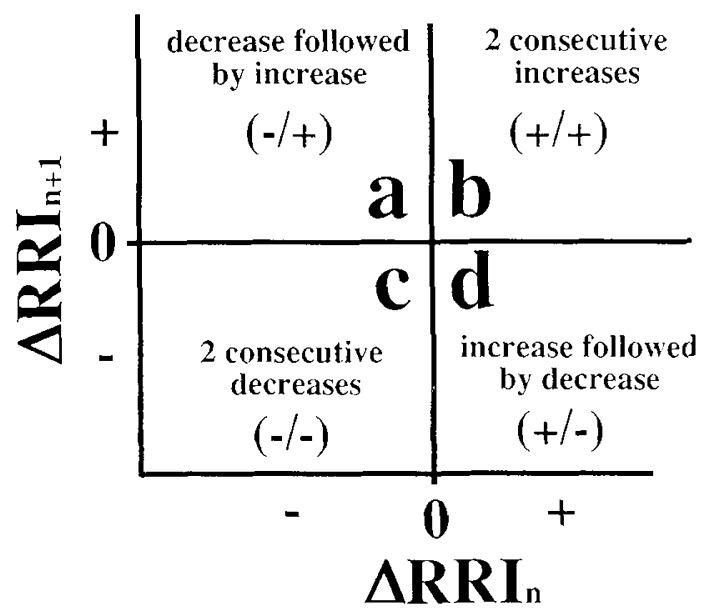

Fig. 1. Diagram of a $\Delta R R_{n+1} v s \Delta R R_{n}$ plot. The notations in each of the quadrants explain the changes in interval lengths represented by each quadrant. For example, a point in quadrant $a$ represents a decrease in interval length [i.e. the difference obtained by subtracting one interval from the previous interval is negative $(-)]$ followed by an increase in interval length [the next difference is positive $(+)$ ]. number of points, resulting either from differences in amount of time spent in each state or from differences in heart rate (in a given time period, an infant with a faster heart rate will show a greater number of $R-R$ intervals than one with a slow heart rate), the total number of intervals in each series was used as a covariate in this analysis.

Analysis of Heart Rate Dependency. Basal heart rate is known to be strongly correlated with several aspects of heart rate variation (19). Inasmuch as previous studies have shown heart rate to be elevated in infants who succumb to $\operatorname{SIDS}(1,2)$, all analyses were repeated using basal heart rate during each sleep-waking state as covariates to determine the heart rate dependence of each finding.

\section{RESULTS}

Summary statistics. Summary statistics for these recordings are presented in Table 1 . Heart rate was higher (indicated by shorter R-R intervals in Table 1 ) in the SIDS victims than in the control infants across all sleep-waking states $(p<0.05)$. This result confirms earlier findings of increased heart rate in SIDS victims during all sleep-waking states $(1,2)$.

The SD of R-R intervals showed a significant risk group $\times$ sleep state interaction $(p<0.05)$; analysis of simple effects indicated that $\mathrm{SD}$ of $\mathrm{R}-\mathrm{R}$ intervals were significantly reduced in the SIDS victims relative to the controls during the AW state only. This finding is also consistent with earlier findings of reduced overall heart rate variation (assessed by interquartile range of R-R intervals) in SIDS victims during the AW state only (2).

$R R_{n+1}$ versus $R R_{\mathrm{n}}$ (Poincaré) plots. Poincaré plots calculated from each sleep-waking state for a control infant and an infant who succumbed to SIDS are shown in Figure 2. The dominant characteristic of the scatter of points in SIDS victims is a relative loss in the "fanning" of the distribution, i.e. a loss of the increased dispersion of succeeding intervals as the cardiac interval lengthens (Fig. 2, Control). In SIDS victims, the scatter showed minimal change in dispersion as intervals increased (rate slowed) (Fig. 2, Control versus SIDS). All Poincaré plots showed a strong beatto-beat correlation; $r$ values ranged from 0.61 to 0.99 (means: $0.87,0.93$, and 0.92 for QS, REM sleep, and AW, respectively), and correlations were significant in all 78 series.

Several aspects of the Poincare plots could be assessed. The principal value of the Poincare plots lies in the demonstration of one interval-next interval dispersion at particular heart rates and the change in dispersion from high to low heart rates. Thus, we examined the point dispersion (range of $y$ values, excluding the extremes) at two distinct values of $x$ : the 10th and 90th percentile values of $x$ (high and low heart rates, respectively). These values represent measures of the range of cardiac intervals after relatively short (fast heart rate) and relatively long (slow heart rate) $\mathrm{R}-\mathrm{R}$ intervals.

Across all sleep-waking states, the SIDS victims showed a significant reduction in point dispersion at both high and low (90 and 10\%) heart rates relative to the controls $(p<0.05)$. ANOVA also showed an interaction of SIDS risk and heart rate. Analysis of simple effects demonstrated that the reduction in point dispersion in SIDS victims relative to controls was more

Table 1. Means (and SEM) of $R-R$ intervals and SD of

\begin{tabular}{|c|c|c|c|}
\hline & QS & REM & $\mathrm{AW}$ \\
\hline \multicolumn{4}{|c|}{ Mean $R-R$ interval } \\
\hline Controls & $478.5(16.6)^{*}$ & $455.9(12.2)^{*}$ & $379.9(8.2)^{*}$ \\
\hline SIDS & $459.2(12.2)^{*}$ & $431.0(9.5)^{*}$ & $352.7(7.9)^{*}$ \\
\hline \multicolumn{4}{|l|}{$\mathrm{SD}$} \\
\hline Controls & $32.2(2.7)$ & $48.4(3.1)$ & $37.9(2.2)^{*}$ \\
\hline SIDS & $34.2(3.6)$ & $45.2(2.9)$ & $31.9(1.2)^{*}$ \\
\hline
\end{tabular}

${ }^{*}$ Differences significant at $p<0.05$. 
Control

SIDS

QS
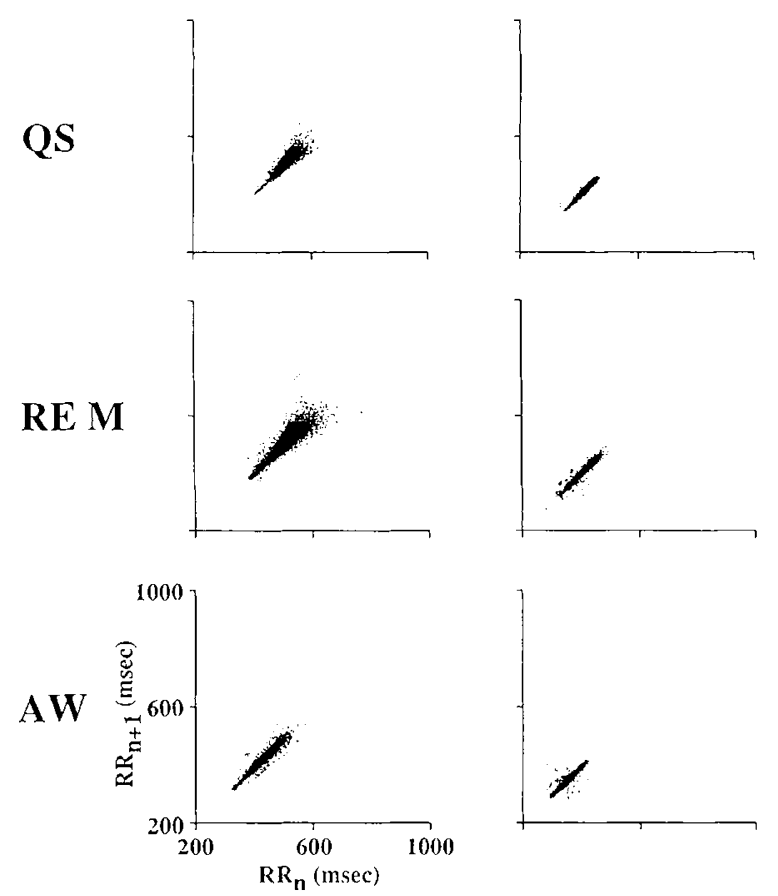

Fig. 2. Poincaré plots of cardiac R-R intervals $\left(R R_{n+1}\right) v s$ the previous intervals $\left(R R_{n}\right)$ for an infant who subsequently succumbed to SIDS and a matched control infant during QS, REM sleep, and AW. Note the tendency for greater dispersion in one interval-next interval with larger $\mathrm{R}-\mathrm{R}$ intervals in the control infant and the relative absence of that tendency in the SIDS victim. Overall variance, which can be assessed by the total range of scatter on both $x$ and $y$ axes, is little affected in this victim of SIDS. All figures are drawn to the same scale.

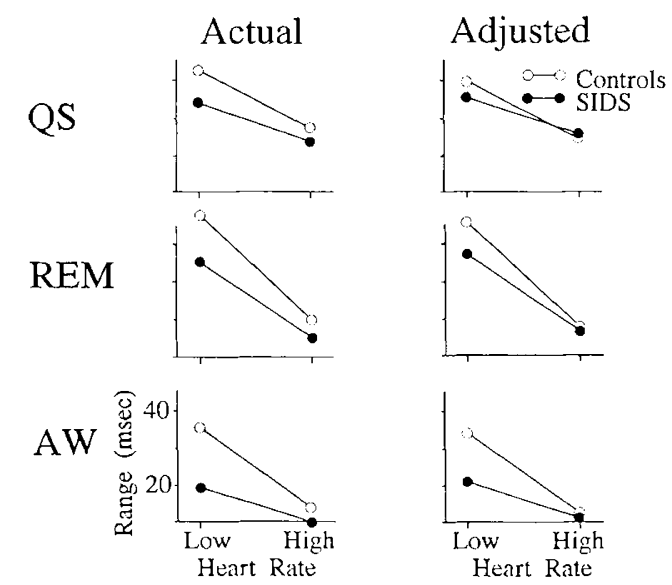

Fig. 3. Mean point dispersion at low (90th percentile of R-R intervals) and high (10th percentile of $R-R$ intervals) heart rates in SIDS victims and controls during QS, REM sleep, and AW before and after the effects of heart rate are partitioned. The raw values (Actual) show a reduction in next-interval dispersions at both low and high heart rates in SIDS victims relative to controls; this difference is significant over all sleepwaking states. When heart rate is introduced as a covariate (Adjusted), the reduction in next-interval dispersion at high heart rates is virtually eliminated; however, at low heart rates, the SIDS victims continue to show reduced interval dispersion relative to controls.

pronounced at low heart rates (long R-R intervals) than at high heart rates. When we corrected for differences in basal heart rate, the simple effect of risk disappeared $(p>0.05)$; however, the interaction between SIDS risk and heart rate remained significant $(p<0.05)$. Figure 3 (Actual) illustrates the mean point dispersion at high (10th percentile of R-R intervals) and low (90th percentile of $\mathrm{R}-\mathrm{R}$ intervals) heart rates in SIDS victims and controls. Figure 3 (Adjusted) demonstrates the same values after correcting for differences in heart rate. The reduction in point dispersion at high heart rates is virtually eliminated by adjusting for differences in basal heart rate; however, at low heart rates, the SIDS victims continue to show reduced point dispersion relative to controls.

ANOVA on the $r$ values for the correlations of one R-R interval with the next showed a significant simple effect of sleepwaking state, with correlations between one interval and the next being significantly lower in QS than in either REM sleep or AW $(p<0.05)$. The ANOVA also demonstrated a significant SIDS risk effect, with the SIDS victims showing stronger correlations than controls across all sleep-waking states $(p<0.05)$. When we corrected for differences in heart rate, the risk effect was reduced to below the significance level $(p>0.05)$.

$\Delta R R_{\mathrm{n}+1}$ versus $\Delta R R_{\mathrm{n}}$ plots. To assess changes in $\mathrm{R}-\mathrm{R}$ intervals without interference from the strong, positive, linear correlations, we plotted $\Delta R_{n+1}$ versus $\Delta R_{n}$ values (Fig. 4 ). In both the SIDS victims and the controis, quadrants $a$ and $d$ (the two quadrants representing alternating increasing and decreasing intervals) contained significantly more points than did quadrants $b$ and $c$ (those quadrants indicating sustained changes in heart rate) across all sleep states $(p<0.05)$.

The principal finding was an interaction between state, risk, and quadrant $(p<0.05)$. In the AW state, the SIDS victims exhibited a substantial decrease in points in the two quadrants reflecting sustained changes in heart rate (quadrants $b$ and $c$ ) relative to controls; this finding was independent of heart rate differences between the groups. Although the SIDS victims also showed fewer time differences greater than $4 \mathrm{~ms}$ in the two quadrants reflecting alternating increases and decreases in heart rate (quadrants $a$ and $d$ ), the difference was significantly more pronounced for quadrants $b$ and $c[p<0.05$ (Fig. 4)]. The sum of points in quadrants $b$ and $c$ (sustained increases greater than $4 \mathrm{~ms} /$ heart beat and sustained decreases greater than $4 \mathrm{~ms} /$ heart beat) during AW for each SIDS victim and each control are plotted against the age of the infant at the time of recording in Figure 5

ANOVA also indicated a simple effect for sleep-waking state. In both groups of infants, the number of R-R interval differences above the minimum of $4 \mathrm{~ms}$ was reduced in all quadrants in $\mathrm{AW}$ relative to the two sleep states $(p<0.05)$.

\section{DISCUSSION}

The procedures used here identified differences between infants who died of SIDS and control infants during sleep where

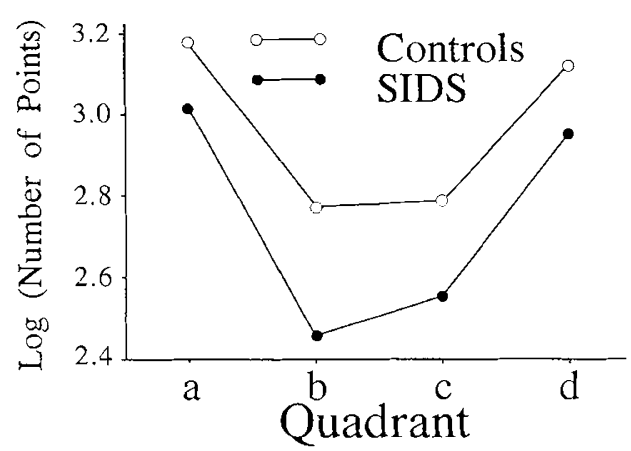

Fig. 4. Mean number of points in each quadrant in SIDS victims (filled circles) and controls (open circles). The common log of each value was used in the computation of the means because the distribution of points was not normal. Quadrant $a$ represents a decrease in R-R interval length followed by an increase; quadrant $b$, two successive increases; quadrant $c$, two successive decreases; and quadrant $d$, an increase followed by a decrease. The SIDS victims showed fewer points than controls in all quadrants; however, the reduction was significantly more profound for quadrants $b$ and $c$ (the two quadrants reflecting sustained changes in $R-R$ interval length). 


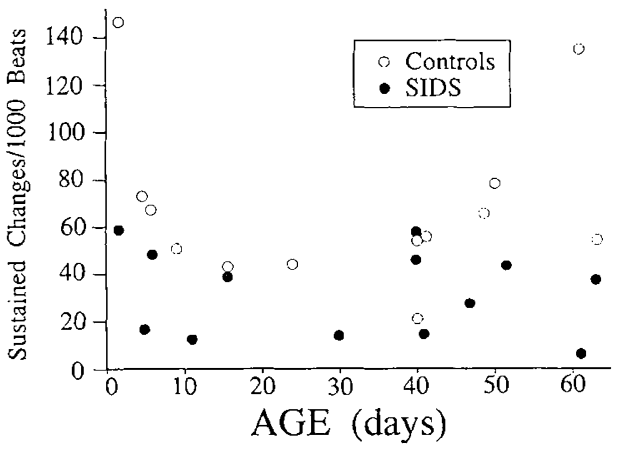

Fig. 5. Number of sustained changes in heart rate (points in quadrants $b$ and $c$ in the $\Delta R_{n+1}$ is $\Delta R R_{n}$ plot) for 13 infants who subsequently succumbed to SIDS (filled circles) and 13 matched control infants (open (ircles). The ordinate represents the number of total points in quadrants $b$ and $c$ combined, and the abscissa represents the age of the infant at the time of the recording. Note that the SIDS victims have significantly fewer points in quadrants $b$ and $c$, reflecting fewer changes in heart rate sustained over three beats, than the control infants.

summary measures failed. Heart rate variability, as assessed here by $\mathrm{SD}$ of R-R intervals and previously by interquartile range of $R-R$ intervals (4), showed differences in AW but not during states of sleep. Instantaneous changes in cardiac intervals measured here, however, differed during sleep as well as AW. Specifically, the dispersion of points at both high and low heart rates in Poincare plots was significantly reduced in SIDS victims relative to control infants across all sleep-waking states. SIDS victims also had stronger correlations of one cardiac R-R interval length with the next than controls during all states. However, some differences between SIDS victims and controls were apparent only during AW. Fewer changes occurred in interbeat intervals during AW. Sustained changes in heart rate (two consecutive changes in the same direction) showed the greatest reduction in SIDS victims. Some of these differences between SIDS victims and controls were independent of differences in heart rate.

Significance of heart rate dependencies. Because some of these differences parallel changes in heart rate and others do not, more than one mechanism may underlie the differences between SIDS victims and controls. In SIDS victims, the dispersion of subsequent intervals is restricted at both high and low heart rates (10th and 90th percentile values of R-R intervals, respectively). Two factors appear to contribute to this effect. The restricted range of next intervals after a short R-R interval (high heart rate) disappeared when we corrected for differences in heart rate; thus, this difference in interval dispersion likely results from physiologic mechanisms similar to those responsible for the increased heart rate in SIDS victims relative to controls. The restricted range of next intervals after longer R-R intervals (low heart rate) in SIDS victims, however, is independent of differences in heart rate and most likely results from another mechanism. Similarly, differences in the number of interval changes were also unaffected by the correction for heart rate. Thus, these differences also appear to be independent of mechanisms that alter heart rate.

The reduction in next-interval dispersion at both high and low basal heart rates indicates that, for a given heart rate, the range of potential R-R interval lengths reflected in subsequent beats is restricted in SIDS victims. Restriction of short-term heart rate variation, as assessed by SD and spectral analytic procedures, is characteristic of reduced vagal tone in adults and animals (2023 ). Thus, a reduction in vagal tone may underlie a portion of the diminution in heart rate variation in victims of SIDS; however, the finding that part of the difference is independent of differences in basal heart rate indicates that another mechanism may be operating.

Patterns of heart rate change. In both SIDS victims and control infants, $\Delta R R_{n+1}$ versus $\Delta R R_{n}$ plots showed a greater number of points in the quadrants representing high-frequency changes in heart rate than in those depicting low-frequency changes. Heart rate variation at the respiratory frequency (respiratory sinus arrhythmia) and lower frequency heart rate variation undoubtedly contribute to the activity in the quadrants reflecting rapid changes in direction $(a$ and $d$ ), but the preponderance of points in these quadrants, relative to the quadrants representing sustained changes in heart rate ( $b$ and $c$ ), indicates that many of these points represent very high frequency heart rate variation with periods of only two interbeat intervals. This very high frequency variation is considerably faster than the respiratory rate, and therefore cannot result from respiratory sinus arrhythmia. The variation more likely represents momentary changes from baseline followed by immediate return to that rate, as if the values were "clamped" about the mean rate.

During AW, the infants who died of SIDS had fewer total successive differences, both greater than $4 \mathrm{~ms}$, than did those who survived infancy. Moreover, the reduction in the number of values in those quadrants representing increases and decreases in heart rate sustained over three beats was proportionally greater than the reduction in very brief changes. In effect, a change in heart rate was more likely to be followed by a change in the opposite direction (a return to baseline) in the SIDS victims than in the controls. The findings suggest that, in the SIDS victims, a larger proportion of heart rate changes may result from momentary deviations from a clamped rate rather than coordinated physiologic responses, which would be sustained over several R$\mathrm{R}$ intervals. The SIDS victims' results suggest that their cardiac interbeat intervals were more resistant to modulation than those of controls.

If changes in heart rate were random and independent, points would be randomly distributed in all four quadrants. The negative correlation between one change in heart rate and the next can be caused only by changes in heart rate that last for one beat and then return to baseline. The return to baseline in so many cases is not random, but suggests active control. We emphasize that these results do not indicate fixed heart rate in the SIDS victims.

In addition to differentiating SIDS victims from other infants, the number of sustained changes showed a profound maturational effect in the control infants. As demonstrated in Figure 5, the number of sustained changes showed a U-shaped curve, starting at a very high level at $2 \mathrm{~d}$ of age, decreasing over the first week or two of life, and then increasing. With only two exceptions, all of the control infants closely followed this curve. This pattern is not unexpected, inasmuch as both heart rate and overall heart rate variation show similar developmental patterns [heart rate increases rapidly over the first week of life, then decreases, and overall heart rate variation decreases in the early postnatal period and increases thereafter $(2,4)]$. It is interesting, however, that the SIDS victims do not show this developmental pattern of sustained changes, although they do show the characteristic patterns of maturation of heart rate and overall heart rate variation $(2,4)$. Furthermore, although some SIDS victims showed almost as many sustained changes as the controls, another subset of the SIDS victims showed a profound reduction in these values (Fig. 5); thus, the population of SIDS victims does not appear to be uniform on this measure.

Autonomic nervous system influences. Cardiac instability, leading to potentially lethal arrhythmia, has been proposed as a mechanism for SIDS deaths (24-27), although the evidence for this cardiac hypothesis is largely circumstantial (28). Hyperactivity or left/right imbalance of cardiosympathetic nerves reduces the electrical stability of the heart (29-31) and may precipitate ventricular fibrillation and sudden cardiac death in adults and animals (32). The cardiac instability hypothesis suggests that during the period of rapid sympathetic nervous system development, which coincides with the period of maximal risk of SIDS, some infants (particularly those with accelerated or asymmetrical sympathetic nervous system development) may be at increased risk of sudden cardiac death. Delayed or deficient cardiovagal 
development may accentuate the arrhythmogenic potential (33, 34).

The parasympathetic nervous system directly mediates shortterm heart rate variation, as well as contributes to lower frequency variation $(35,36)$; thus, decreased vagal tone may contribute to the reduced beat-to-beat heart rate variation in SIDS victims. It is unlikely, however, that overall diminished vagal activity underlies all of the findings here. Heart rates in infants who later succumbed were higher than those of controls, but the SIDS victims retained a wide range of slow, as well as more rapid, heart rates. Furthermore, increased sympathetic activation can reduce short-term heart rate variation by inhibiting vagal activation of the heart $(37,38)$. Thus, either sympathetic hyperactivity or parasympathetic hypoactivity, or some interaction of the two, may underlie the diminished beat-to-beat heart rate variation observed in SIDS victims.

The reduction in interbeat dispersion at high heart rates is related to differences in basal heart rate, whereas the reduction at low heart rates is not, suggesting that different mechanisms underlie the reduction in beat-to-beat variation at high and low heart rates. The clamping of cardiac intervals, with the vast majority of heart rate changes being "corrected" by the successive beat, suggests active vagal mechanisms, because sympathetic mechanisms act too slowly to affect such rapid adjustments (39, 40). However, sustained, high- and mid-frequency variation, which is principally under the control of parasympathetic influences, was diminished in infants who succumbed; this finding suggests a less "reactive" vagal system. Thus, infants who subsequently die of SIDS may suffer from both increased sympathetic tone and impaired vagal tone.

A number of limbic forebrain structures project to brainstem autonomic control regions, and recent evidence suggests that these rostral structures can modulate autonomic control (41-44). Thus, disturbances in the functional integrity of forebrain influences may underlie some of the differences in cardiac dynamics observed in these infants who succumbed to SIDS. Delayed myelination of limbic pathways has been described in victims of SIDS (45). A functional disruption of limbic forebrain pathways induced by such a maturational delay might contribute to the finding described here.

In addition to demonstrating reduced heart rate variability in infants who succumb to SIDS, we described the types of variation encountered at low and high rates and the changes in interval lengths at different heart rates. Such descriptions are useful in assessing cardiac patterns in congestive heart failure patients who are at risk for sudden death (8). Determination of changes in heart intervals at different rates may be useful in assessing the particular physiologic mechanisms underlying SIDS.

Reduced heart rate variation is correlated with risk of cardiac arrhythmia (including ventricular fibrillation) and sudden cardiac death in adults and dogs with myocardial damage (46-48). The reduction in heart rate variability demonstrated in this and previous studies of these SIDS victims $(3,4)$ may also indicate a potential for cardiac instability. However, SIDS victims seldom show myocardial damage (49). Myocardial damage is not a prerequisite for sudden cardiac death, but, understandably, there is little evidence on the relationship between heart rate variability and sudden death in the absence of such pathology. Thus, the potential relationship between diminished heart rate variation and risk of cardiac arrhythmia in SIDS victims remains unclear.

Limitations. It should be noted that the use of the automated infant sleep state classification system introduces a potentially confounding variable. The state classification system, which was developed based on normal infants, uses measures of cardiac rate and variation in addition to respiratory measures. In normal infants, this automated infant sleep-state classification system achieves a level of accuracy similar to that of a trained observer using all physiologic signals (15). Some of the cardiac measures it uses, however, are altered in infants at risk for SIDS. Both cardiac and respiratory measures are normalized within each recording before sleep state classification; thus, differences in mean heart rate and variation in the SIDS victims could not have affected the assignment of sleep-waking states. Differences in the way that these factors are modulated by state may, however, reduce the accuracy of the classification system in pathologic cases.

Because the state classification system is based partially on cardiac measures, some of which may be associated with the measures of interest in this study, we examined differences in heart rate dynamics across sleep-waking states after using other measures of cardiac function to define those states. This implementation was considered a necessary evil, inasmuch as respiratory measures alone differentiate REM sleep and AW with reduced accuracy, particularly during the first month of life.

The findings demonstrate abnormalities in the beat-to-beat dynamics of cardiac control in infants who later die of SIDS. Some of these differences parallel changes in heart rate and may result from similar physiologic mechanisms as those responsible for the heart rate changes. Several aspects of cardiac dynamics found in the risk group, however, were unrelated to differences in basal heart rate, indicating that at least a second physiologic or anatomical mechanism contributes to the findings described here. A portion of the differences between groups appears to reflect cardiac patterning that is actively clamped, causing heart rate to be relatively unresponsive to physiologic mechanisms. Sustained variation (two or more sustained changes in the same direction) appears to be lacking in infants who later die. The finding that this diminution in reactivity is enhanced during AW suggests a loss of integrative neural mechanisms particularly active during that state. Although it is not clear that cardiac instability is a factor in SIDS deaths, the changes in dynamic patterns of cardiac rate reported here indicate pronounced alterations in autonomic control of the heart in infants who succumb to SIDS. These aberrations may be indicative of failure in other physiologic systems that may interfere with vital functions.

\section{REFERENCES}

1. Wilson AJ, Stevens V, Franks CI, Alexander J, Southall DP 1985 Respiratory and heart rate patterns in infants destined to be victims of the sudden infant death syndrome: average rates and their variability measured over 24 hours. Br Med J 290:497-501

2. Schechtman VL, Harper RM, Kluge KA, Wilson AJ, Hoffman HJ, Southall DP 1988 Cardiac and respiratory patterns in normal infants and victims of the sudden infant death syndrome. Sleep 11:413-424

3. Kluge KA, Harper RM, Schechtman VL, Wilson AJ, Hoffman HJ, Southall DP 1988 Spectral analysis assessment of respiratory sinus arrhythmia in normal infants and infants who subsequently died of sudden infant death syndrome. Pediatr Res 24:677-682

4. Schechtman VL, Harper RM, Kluge KA, Wilson AJ, Hoffman HJ, Southall DP 1989 Heart rate variation in normal infants and victims of the sudden infant death syndrome. Early Hum Dev 19:167-181

5. Schechtman VL, Harper RM, Kluge KA, Wilson AJ Southall DP 1990 Correlations between cardiorespiratory measures in normal infants and victims of the sudden infant death syndrome. Sleep 13:304-317

6. Valimaki IAT, Nieminen T, Antila KJ, Southall DP 1988 Heart rate variability and SIDS: examination of heart-rate patterns using an expert system generator Ann NY Acad Sci 533:228-237

7. Schechtman VL, Harper RM, Wilson AJ, Southall DP 1991 Sleep apnea in infants who succumb to the sudden infant death syndrome. Pediatrics 87:841-846

8. Woo MA, Stevenson WG, Moser DK, Trelease RB, Harper RM 1992 Pattern of beat-to-beat heart variability in advanced heart failure. Am Heart $J$ 123:704-710

9. Woo MS, Woo MA, Gozal D, Jansen MT, Keens TG, Harper RM 1992 Heart rate variability in congenital central hypoventilation syndrome. Pediatr Res 31:291-296

10. Skinner JE, Carpeggiani C, Landisman CE, Fulton KW 1991 Correlation dimension of heartbeat intervals is reduced in conscious pigs by myocardial ischemia. Circ Res 68:966-976

11. Garfinkel AJ, Raetz SL, Harper RM 1992 Heart rate dynamics after acute cocaine administration. J Cardiovasc Pharmacol 19:453-459

12. Abraham R, Shaw C 1983 Dynamics: The Geometry of Behavior Part 2 Chaotic Behavior. Aerial Press, Santa Cruz, CA

13. Harper RM, Hoppenbrouwers T, Sterman MB, McGinty DJ, Hodgman J 1976 Polygraphic studies of normal infants during the first six months of life. I. Heart rate and variability as a function of state. Pediatr Res 10:945-95i

14. Southall DP, Johnston F, Shinebourne EA. Johnston PGB 1981 A 24-hour 
electrocardiographic study of heart rate and rhythm patterns in a population of healthy children. Br Heart J 45:281-291

15. Harper RM, Schechtman VL, KJuge KA 1987 Machine classification of infant sleep state using cardiorespiratory measures. Electroencephalogr Clin Neurophysiol 67:379-387

16. Schechtman VL, Kluge KA, Harper RM 1988 Time-domain system for assessing variation in heart rate. Med Biol Eng Comput 26:367-373

17. Anderson TW (ed) 1958 An Introduction to Multivariate Statistical Analysis. Wiley, New York

18. Huynh H, Feldt LS 1976 Estimation of the box correction for degrees of freedom from sample data in the randomized block and split-plot designs. $J$ Educ Statist 1:69-82

19. Mazza NM, Epstein MAF, Haddad GG, Law HS, Mellins RB, Epstein RA 1980 Relation of beat-to-beat variability to heart rate in normal sleeping infants. Pediatr Res 14:232-235

20. Chipps DR, Kraegen EW, Zelenka GS, McNamara ME, Chisholm DJ 1981 Cardiac beat to beat variation: age related changes in the normal population and abnormalities in diabetics. Aust NZ J Med 11:614-620

21. Katona PG, Jih F 1975 Respiratory sinus arrhythmia: noninvasive measure of parasympathetic cardiac control. J Appl Physiol 39:801-805

22. Lishner M, Akselrod S, Mor Avi V, Oz O, Divon M, Ravid M 1987 Spectral analysis of heart rate fluctuations. A non-invasive, sensitive method for the early diagnosis of autonomic neuropathy in diabetes mellitus. J Auton Nerv Syst 19:119-125

23. Pagani $M$, Malfatto $G$ Pienini S, Casati R, Masu AM, Poli M, Guzzetti S, Lombardi F, Cerutti S, Malliani A 1988 Spectral analysis of heart rate variability in the assessment of autonomic diabetic neuropathy. J Auton Nerv Syst 23:143-153

24. Schwartz PJ 1976 Cardiac sympathetic innervation and the sudden infant death syndrome: a possible pathogenetic link. Am J Med 60:167-172

25. Schwartz PJ, Segantini A 1988 Cardiac innervation, neonatal electrocardiography, and SIDS: a key for a novel preventive strategy? Ann NY Acad Sci 533:210-220

26. Froggatt $P$, James TN 1973 Sudden unexpected death in infants: evidence of a lethal cardiac arrhythmia. Ulster Med J 42:136-152

27. Froggatt $P 1977$ A cardiac cause in cot death: a discarded hypothesis? J Ir Med Assoc 70:408-414

28. Guntheroth WG 1989 Theories of cardiovascular causes in sudden infant death syndrome. J Am Coll Cardiol 14:443-447

29. Corr PB, Yamada KA, Witkowski FX 1986 Mechanisms controlling cardiac autonomic function and their relation to arrhythmogenesis. In: Fozzard HA Haber E, Jennings RB, Katz AM (eds) The Heart and the Cardiovascular System. Raven Press, New York, pp 1343-1401

30. Schwartz PJ, Vanoli E 1981 Cardiac arrhythmias elicited by interaction between acute myocardial ischemia and sympathetic hyperactivity: a new experimental model for the study of antiarrhythmic drugs. J Cardiovasc Pharmacol 3:1251-1259

31. Schwartz PJ, Billman GE, Stone HL 1984 Autonomic mechanisms in ventricular fibrillation induced by myocardial ischemia during exercise in dogs with a healed myocardial infarction. An experimental preparation for sudden cardiac death. Circulation 69:780-790
32. Schwartz PJ, Stone HL 1982 The role of the autonomic nervous system in sudden coronary death. Ann NY Acad Sci 382:162-180

33. Schwartz PJ, Stone HL 1985 The analysis and modulation of autonomic reflexes in the prediction and prevention of sudden death. In: Zipes DP, Jalife J (eds) Cardiac Electrophysiology and Arrhythmias. Grune \& Stratton, New York, pp 165-176

34. Schwartz PJ 1987 The quest for the mechanisms of the sudden infant death syndrome: doubts and progress. Circulation 75:677-683

35. Akseirod S, Gordon D, Ubel FA, Shannon DC, Barger AC, Cohen RJ 198 Power spectrum analysis of heart rate fluctuation: a quantitative probe of beat-to-beat cardiovascular control. Science 213:220-222

36. Randall DC, Brown DR, Raisch RH, Yingling JD, Randall WC 1991 SA nodal parasympathectomy delineates autonomic control of heart rate power spectrum. Am J Physiol 260:H985-H988

37. Warner MR, Levy MN 1989 Neuropeptide $Y$ as a putative modulator of the vagal effects on heart rate. Circ Res 64:882-889

38. Hall GT, Potter EK 1990 Attenuation of vagal action following sympathetic stimulation is modulated by prejunctional $\alpha_{2}$-adrenoceptors in the dog. $J$ Auton Nerv Syst 30:129-138

39. Levy NM, Martin PJ 1979 Neural control of the heart. In: Berne RM Sperelakis N, Geiger SR (eds) Handbook of Physiology: Section 2. The Cardiovascular System. American Physiological Society, Bethesda, MD, pp $581-620$

40. Karemaker JM 1985 Cardiac cycle time effects: information processing and the latencies involved. In: Orlebeke JF, Mulder G, van Doornen LJP (eds) Psychophysiology of Cardiovascular Control: Models, Methods, and Data. Plenum Press, New York, pp 535-548

41. Buchanan SL, Powell DA 1982 Cingulate cortex: its role in Pavlovian conditioning. J Comp Physiol Psychol 96:755-774

42. Buchanan SL. Powell DA 1982 Cingulate damage attenuates conditioned bradycardia. Neurosci Lett 29:261-268

43. Zhang JX, Harper RM, Ni H 1986 Cryogenic blockade of the central nucleus of the amygdala attenuates aversively conditioned blood pressure and respiratory responses. Brain Res 386:136-145

44. Frysinger RC, Harper RM 1989 Cardiac and respiratory correlations with unit discharge in human amygdala and hippocampus. Electroencephalogr Clin Neurophysiol 72:463-470

45. Kinney HC, Brody BA, Finkelstein DM, Vawter GF, Mandell F, Gilles FH 1991 Delayed central nervous system myelination in the sudden infant death syndrome. J Neuropath Exp Neurol 50:29-48

46. Kreiger RE, Miller J, Bigger Jr JT, Moss AJ, Multicenter Post-Infarction Research Group 1987 Decreased heart rate variability and its association with increased mortality after acute myocardial infarction. Am J Cardio 59:256-262

47. Martin GJ, Magid NM, Myers G, Barnett PS, Schaad JW, Weiss JS, Lesch M, Singer DH 1987 Heart rate variability and sudden death secondary to coronary artery disease during ambulatory electrocardiographic monitoring. Am J Cardiol 60:86-89

48. Hull Jr SS, Evans AR, Vanoli E, Adamson PB, Stramba-Badiale M, Albert DE, Foreman RD, Schwartz PJ 1990 Heart rate variability before and after myocardial infarction in conscious dogs at high and low risk of sudden death. J Am Coll Cardiol 16:978-985

49. Beckwith JB 1973 The sudden infant death syndrome. Curr Probl Pediatr 3:336 